\title{
Persistent candidemia in very low birth weight neonates: risk factors and clinical significance
}

\author{
Jinjian $\mathrm{Fu}^{1{ }^{*}}$, Yanling Ding ${ }^{1+}$, Yongjiang Jiang ${ }^{2 \dagger}$, Shengfu $\mathrm{Mo}^{1+}$, Shaolin $\mathrm{Xu}^{1}$ and Peixu Qin ${ }^{1}$
}

\begin{abstract}
Background: The prevalence and risk factors for persistent candidemia among very low birth weight infants are poorly understood. This study aimed to investigate the epidemiology of persistent candidemia over a 4-year period in a neonatal intensive care unit (NICU) in Liuzhou, China.

Methods: We retrospectively extracted demographic data, risk factors, microbiological results and outcomes of very low birth weight infants with candidemia in our hospital between January 2012 and November 2015. Persistent candidemia was defined as a positive blood culture for $>5$ days. Logistic regression was used to identify risk factors associated with persistent candidemia.

Results: Of 48 neonates with candidemia, 28 had persistent candidemia. Both mechanical ventilation and intubation were significantly associated with increased rates of persistent candidemia ( $P=0.044$ and 0.004 , respectively). The case fatality rate for the persistent candidemia group was 14.3\%.

Conclusion: The rate of persistent candidemia was high among very low birth weight neonates. Mechanical ventilation and intubation were the major factors associated with the development of persistent candidemia. This study highlights the importance of intensive prevention and effective treatment among neonates with persistent candidemia.
\end{abstract}

Keywords: Persistent candidemia, Very low birth weight, Neonates, Epidemiology

\section{Background}

Candida species have emerged as important nosocomial infection pathogens associated with significant morbidity and mortality in very low birth weight (VLBW) neonates [1-4]. Candidemia is the third most common nosocomial bloodstream infection during late-onset neonatal sepsis [1]. It affects 10 to $20 \%$ of extremely low birth weight infants and 2 to $16 \%$ of very low birth weight neonates and is responsible for 25 to $30 \%$ of morbidity in neonatal intensive care units (NICUs) [2-7]. It was reported that the infants who survive candidemia frequently have long-term neurodevelopmental impairment, which occurred in 57\% of these high-risk infants [7].

\footnotetext{
* Correspondence: fujinjianaa@126.com

${ }^{\dagger}$ Jinjian Fu, Yan Ling Ding, Yongjiang Jiang and Sheng Fu Mo contributed equally to this work.

${ }^{1}$ Department of Laboratory, Liuzhou Maternity and Child Healthcare Hospital, 50th Yingshan Road, Chengzhong District, Liuzhou 545001, China Full list of author information is available at the end of the article
}

The contributing factors in high-risk groups include prematurity, VLBW, catheter and endotracheal tube use, prolonged NICU stay, broad-spectrum antibiotic use and total parenteral nutrition [8-10]. Lack of specific signs or symptoms in the development of candidemia among VLBW infants results were in a high risk of fatality [8]. Early diagnosis is crucial for infection control and initiating effective treatment.

Although VLBW is a well-known risk factor in the development of candidemia, it is uncertain whether this risk factor also contributes to persistent candidemia or mortality. Only a few studies have evaluated the risk factors and mortality for persistent candidemia in VLBW infants, and the results remain controversial. The potential risk factors and attributable mortality of persistent candidemia in VLBW infants in Western China are unknown. Therefore, we conducted this study to identify the incidence, risk factors, microbiological results and

(c) The Author(s). 2018 Open Access This article is distributed under the terms of the Creative Commons Attribution 4.0 International License (http://creativecommons.org/licenses/by/4.0/), which permits unrestricted use, distribution, and 
mortality associated with persistent candidemia in VLBW infants in China.

\section{Methods \\ Data collection}

A retrospective chart review of neonates admitted to the NICU of the Liuzhou Maternity and Child Healthcare Hospital from January 2012 to November 2015 was performed. Infants born at the hospital or transferred to the $\mathrm{NICU}$ at $<5$ days old were included. At least one blood culture obtained by peripheral vein puncture that grew Candida species was defined as candidemia. Based on the study by Levy et al [11], if a single patient had a positive blood culture lasted for $>5$ days starting from the first culture result indicated positive for Candida, the infection was considered persistent candidemia.

An electronic database was used to collect and record data. These included gestational age, birth weight, admission age, gender, delivery type, fetal membrane rupture duration, necrotizing enterocolitis, neurodevelopmental impairment, congenital diseases (such as congenital heart disease, glucose-6-phosphatedehydrogenase deficiency, and thalassemia), incidence of abdominal surgery, mechanical ventilation use, indwelling central venous catheter use, intubation $>6$ days, rescue history, total parenteral nutrition status, hospitalization duration, 3rd generation cephalosporin use, carbapenem use, vancomycin use, antibiotic therapeutic duration, multiple antibiotic use $(\geq 3$ classes), Candida species, and mortality.

\section{Microbiologic methods}

Blood cultures were incubated using the BacT/Alert 3D system (bioMerieux). Candida species were cultured in CHROM Agar medium (bioMerieux) and isolates were identified using API 20C AUX (bioMerieux).

\section{Statistical analysis}

Statistical analysis was carried out using SPSS version 20.0 statistical software (SPSS Inc., Chicago, IL, USA). Potential risk factors associated with persistent candidemia were identified using logistic regression analysis. Variables with 2-tailed $P<0.05$ were defined as statistically significant. Odds ratios (ORs) along with $95 \%$ confidence intervals (CIs) were used to assess the strength of any association.

\section{Results}

\section{Incidence}

During the 4-year period, a total of 5075 infants were admitted to the NICU, of which 484 were VLBW infants. A total of 48 cases among the VLBW infants were diagnosed with candidemia, resulting in a candidemia incidence of 9.5 per 1000 infants. Among the very low birth weight infants, the incidence of candidemia was
9.9\%. For the 48 infants who suffered from candidemia, 28 experienced positive blood culture for $>5$ days and were subsequently reported as having persistent candidemia. The persistent candidemia rate was $5.8 \%$ among VLBW infants.

Among the 48 Candida species, Candida albicans accounted for $39.6 \%$ of all cases (19/48), followed by Candida glabrata at 33.3\% (16/48), and Candida tropicalis at $27.1 \%$ (13/48), no other Candida was found except C.albicans, C. glabrata, and C. tropicalis. C.albicans accounted for $25 \%(7 / 28)$ of cases of persistent candidemia. Non-albicans species were the leading causative pathogens of persistent candidemia, accounting for $75.0 \%$ of all cases, C. glabrata and C. tropicalis accounted for $42.9 \%(12 / 28)$ and $32.1 \%(9 / 28)$ of cases, respectively.

\section{Demographics}

Among the 48 VLBW infants with candidemia, 17 were males and 31 were females. The median admission age was 3.2 days (range: 1.7-4.8 days), the median birth weight was $1154.5 \mathrm{~g}$ (range: $950.2 \mathrm{~g}-1358.8 \mathrm{~g}$ ), and the median gestational age was 29.6 weeks (range: 27.6-31.6 weeks) (Table 1).

\section{Clinical presentation}

Most infants with candidemia received mechanical ventilation $(70.8 \%, 34 / 48)$, central venous catheters (58.3\%, 28/48), total parenteral nutrition $(93.8 \%, 45 / 48)$, and had a rescue history $(66.7 \%, 32 / 48)$ while $48 \%(23 /$ 48) of them received intubation for $>6$ days. All of them received at least one class of antibiotics in the week before candidemia was diagnosed, and $64.6 \%$ received at least three classes of broad-spectrum antibiotics. The median antibiotic therapeutic duration was 38.9 days (range 20.7-58.0 days) while $85.4 \%$ of the neonates received prophylactic antifungal therapy with a median therapeutic duration of 10.4 days (range: 4.3-16.6 days). The median hospital length of stay was 54.9 days (range: 32.6-77.2 days).

\section{Risk factors}

Compared to the non-persistent candidemia group, the persistent candidemia cases had a significantly lower birth weight [1229.0 vs. $1097.8 \mathrm{~g} ; P<0.001]$ and significantly longer antibiotic therapeutic duration [31.1 $(16.6,55.6)$ days vs. $44.5(24.3,64.7)$ days; $P<0.001]$. Persistent candidemia infants also had higher incidence of necrotizing enterocolitis $(32.1 \%$ vs. $5.0 \% ; P<0.001)$, mechanical ventilation $(85.7 \%$ vs. $50.0 \%$; $P<0.001)$, and intubation $(71.4 \%$ vs. $15.0 \% ; P<0.001)$ than the non-persistent cases. The non-albicans species were found more frequently in the persistent candidemia group $(75.0 \%$ vs. $40.0 \% ; P<0.001)$. A logistic regression analysis using 
Table 1 Clinical characteristics of neonates with and without persistent candidemia

\begin{tabular}{|c|c|c|c|c|}
\hline \multirow[t]{2}{*}{ Variable } & \multicolumn{2}{|l|}{ Persistent candidemia } & \multirow[t]{2}{*}{$P$ value } & \multirow{2}{*}{$\begin{array}{l}\text { Odds ratio (OR) } \\
(95 \% \mathrm{Cl})\end{array}$} \\
\hline & Yes mean $(95 \% \mathrm{Cl})$ or $n(\%)$ & No mean $(95 \% \mathrm{Cl})$ or $n(\%)$ & & \\
\hline \multicolumn{5}{|l|}{ Demographics } \\
\hline Gestational age (wks) & $29.6(27.3,31.9)$ & $29.7(28.1,31.2)$ & 0.766 & \\
\hline Birth weight (g) & $1097.8(866.4,1309.2)$ & $1229.0(1051.1,1046.9)$ & 0.029 & \\
\hline Male gender, $n(\%)$ & $12(42.9)$ & $5(25.0)$ & 0.207 & $0.44(0.13-1.57)$ \\
\hline Admission age & $1.3(0.4,2.2)$ & $1.8(2.4,6.0)$ & 0.611 & \\
\hline \multicolumn{5}{|l|}{ Risk factors } \\
\hline Necrotizing enterocolitis & $9(32.1)$ & $1(5.0)$ & 0.046 & $9.0(1.04-78.17)$ \\
\hline Neurodevelopmental impairment & $6(21.4)$ & $6(30.0)$ & 0.501 & $0.64(0.17-2.37)$ \\
\hline Vaginal delivery & $13(46.4)$ & $7(35.0)$ & 0.430 & $1.61(0.49-5.25)$ \\
\hline Fetal membrane rupture (h) & $46.4(25.1,117.7)$ & $18.8(37.5,75.1)$ & 0.143 & \\
\hline Congentital diseases & $15(53.6)$ & $10(50.0)$ & 0.867 & $1.15(0.37-3.64)$ \\
\hline Abdominal surgery & $3(10.7)$ & $0(0.0)$ & - & - \\
\hline Mechanical ventilation & $24(85.7)$ & $10(50.0)$ & 0.011 & $6.00(1.52-23.70)$ \\
\hline Central venous catheter & $15(59.6)$ & $13(65.0)$ & 0.430 & $0.62(0.19-2.03)$ \\
\hline Intubation & $20(71.4)$ & $3(15.0)$ & 0.000 & $14.17(3.24-61.99)$ \\
\hline Total parenteral nutrition & $26(92.9)$ & $19(95.0)$ & 0.764 & $0.68(0.06-8.11)$ \\
\hline Hospitalization duration (d) & $58.8(33.8,93.8)$ & $49.3(32.4,66.2)$ & 0.147 & \\
\hline $3^{\text {rd }}$ cephalosporins use & $16(59.3)$ & $13(65.0)$ & 0.689 & $0.78(0.24-2.59)$ \\
\hline Carbapenems use & $26(92.9)$ & $16(80.0)$ & 0.201 & $3.25(0.53-19.82)$ \\
\hline Vancomycin use & $7(25.0)$ & $2(10.0)$ & 0.203 & $3.00(0.55-16.31)$ \\
\hline Multiple antibiotic use & $18(64.3)$ & $13(65.0)$ & 0.959 & $0.97(0.29-3.22)$ \\
\hline Antibiotic therapeutic duration (d) & $44.5(24.3,64.7)$ & $31.1(16.6,55.6)$ & 0.015 & \\
\hline Non-C.albicans & $21(75.0)$ & $8(40.0)$ & 0.017 & $4.50(1.31-15.52)$ \\
\hline Prophylaxis antifungal therapy & $25(89.3)$ & $16(80.0)$ & 0.375 & $2.08(0.41-10.56)$ \\
\hline Antifungal therapeutic duration (d) & $11.3(4.6,18.0)$ & $9.2(4.6,14.4)$ & 0.243 & \\
\hline \multicolumn{5}{|l|}{ Outcome } \\
\hline Death & $4(14.3)$ & $1(5.0)$ & 0.320 & $3.17(10.33-30.73)$ \\
\hline
\end{tabular}

backward model selection method on the 48 infants showed that candidemia cases on mechanical ventilation had a significantly increased risk of developing into persistent candidemia $(\mathrm{OR}=5.72 ; 95 \% \mathrm{CI}=1.05-31.25)$, while intubation longer 6 days had an even higher risk $(\mathrm{OR}=10.53 ; 95 \% \mathrm{CI}=2.11,52.59)($ Table 2$)$.

\section{Outcome}

The overall mortality among 484 VLBW infants was $10.4 \%$. The mortality for the persistent candidemia group was $14.3 \%$, and for those who experience

Table 2 Multivariate analysis for persistent candidemia

\begin{tabular}{llll}
\hline Risk factor & Odds ratio & $95 \% \mathrm{Cl}$ & $P$ value \\
\hline Intubation & 10.53 & $2.11-52.59$ & 0.004 \\
Mechanical ventilation & 5.72 & $1.05-31.25$ & 0.044 \\
\hline
\end{tabular}

none-persistent candidemia was $5.0 \%$. This difference was not statistically significant $(P=0.320)$.

\section{Discussion}

Candidemia remains a significant cause of morbidity and mortality in premature infants. The incidence of neonatal candidemia in our NICU (9.5 per 1000 infants) was similar to those found in many other studies, which reported incidences of candidemia near 10 per 1000 patient discharges $[2,4,10]$.

It was remarkable that the majority (58.3\%) of VLBW neonatal candidemia cases were persistent candidemia, which was similar to one study from Israel [11] that reported persistent candidemia in 52\% of infants. Hammoud et al [12] identified that $60 \%$ of neonatal blood stream infections were persistent. The definition of persistent candidemia in the current study and the investigation was conducted by Levy et al [11] Although 
Robinson et al [13] reported an incidence of candidemia of $24.3 \%$ in a NICU located in the USA, their assessment of persistent candidemia incidence differed from Levy et al [11], which may explain the difference. The variation incidence highlights the importance of a consistent definition for persistent candidemia in neonates.

Many risk factors have been implicated in the pathogenesis of candidemia in infants, the most consistent one was prematurity, especially in very low birth weight groups $[2,5,7,8]$. The results of the present study indicated that low birth weight was significantly associated with persistent candidemia, as revealed by univariate analysis, although no statistical significance was found by multivariate analysis. This finding was similar to a previous study [12], which also showed that the lower the birth weight, the greater the susceptibility to develop persistent candidemia (median birth weight, 970 and1130 $\mathrm{g}$ for the persistent candidemia and candidemia groups, respectively; $P=0.04$ ). VLBW infants may have a higher risk of developing persistent candidemia because of their immature immune system, which may lead to an inability to eliminate pathogens from the bloodstream at the initiation of antifungal therapy.

Previous studies reported that C.albicans was the most frequent cause of candidemia in neonates, followed by C. parapsilosis [11]. Our study indicated that C.albicans was the predominant Candida species in VLBW infants, followed by Non-albicans such as C. glabrata and C. tropicalis. This pattern was consistent with previous reports conducted by Chinese, the American and Australian neonatal research groups [7, 9, 14-17], suggesting different epidemiology for neonatal candidemia around the world. A significantly higher frequency of non-albicans species (42.9 and 32.1\% for C.glabrata and C.tropicalis, respectively) was found among VLBW infants with persistent candidemia compared to C.albicans $(25.0 \%)$ in the current study. Published data addresses the significance of widespread implementation of prophylactic or empirical antifungal therapy in NICUs and PICUs [14-19], which may change Candida ecology. The identified risk factor for C.glabrata infection may be the result of broad-spectrum antibiotic use, particularly anti-aerobic and azole use as the empirical coverage for Gram-negative, Gram-positive and Fungal organisms in pediatric populations $[2,9,16,19]$. One previous study identified C.glabrata as a risk factor for the development of persistent candidemia [20]. Additionally, non-albicans species themselves may also play a critical role in the pathogenesis of persistent candidemia due to their notorious capabilities of adherence to foreign surfaces, high virulence, and inherent or potential resistance to fluconazole [2, 3, 9, 19, 21-23].

Antibiotic exposure and, more importantly, the choice of an anti-aerobic spectrum of activity for routine prophylaxis or empirical therapy was the strongest risk factor for candidemia [14, 16, 24]. Our study was consistent with a previous report which showed that receipt of broad-spectrum antibiotics in the 7 days prior to candidemia diagnosis was a strong risk factor in VLBW infants [25]. A significantly longer duration of preceding antibiotic use was observed among infants with persistent candidemia when compared to infants with one episode of candidemia in the current study. This was consistent with the findings of a USA-based neonatal research group, which showed that prolonged antibiotic exposure was associated with persistent candidemia [26]. It is remarkable that the majority of infants in this study received empirical therapy $(87.5 \%)$ in the preceding month. Moreover, over $85.4 \%$ of infants received multiple classes of antibiotics, either concomitantly or sequentially. Our data was consistent with the study that confirmed antibiotic prophylaxis was a common practice in many NICUs in China and around the world $[9,16,17,19,27]$. This finding highlights the need to reduce antibacterial exposure and employ effective medical practices for infection control.

Multiple explanations for the development of candidemia in neonatal groups have been suggested, and the most convincing explanation was the use of medical catheters and mechanical ventilation. Invasive operations provide a portal of entry and adhesion for Candida species and may be responsible for horizontal transmission [28]. It was suggested that due to the Candida species' ability to adhere to foreign materials, a residual fungal deposit might exist even after prompt removal of infected catheters or medical appliances; thus, it may take some time to eliminate the Candida species given that the antifungal therapy is effective [29]. Medical appliances, such as intubation tubes, may be the cause of persistent candidemia [18]. In the current study, both mechanical ventilation and intubation were significantly associated with persistent candidemia, as revealed by univariate and multivariate regression analysis. Previous studies have also shown that medical catheter retention increased the risk of candidemia death [30] (OR, 95\% CI $=2.50,1.06-5.91)$, and early removal of catheters significantly reduced candidemia-associated mortality (OR, 95\% CI $=20.5,3.9-106.5$ ), suggesting that the implementation of appropriate medical practices, such as early removal of intubation, might improve the prognosis of VLBW infants with persistent candidemia.

Candidemia-related mortality rates in neonates range between 43 and 54\% [31,32]. The candidemia mortality rate in our study was $10.4 \%$, and all occurred in the persistent candidemia group, which had a mortality rate of $17.9 \%$. Our report was consistent with the investigation conducted by Levy et al [11], which showed that the 
crude mortality rate in newborn infants was $17.8 \%$. However, some studies showed that persistent candidemia infections have higher mortality rates compared to non-persistent candidemia. There are several explanations about this discrepancy, one being that we failed to identify an apparent source of the persistent candidemia in the majority of cases, as only five catheter tips tested positive for Candida species (three of which had persistent candidemia). Another explanation was that we defined persistent candidemia without considering the effectiveness of the antifungal therapy, which may result in underestimation of the clinical relevance of persistent candidemia.

The present analysis was limited by the retrospective design and the study's location in a single center. Additionally, the small sample size may have compromised the statistical power of the study. A larger, multicenter prospective study is required to identify additional risk factors, ascertain the burden of persistent candidemia, and determine the antifungal susceptibility profiles to help pediatricians employ the proper intensive prevention and treatment practices.

\section{Conclusions}

In conclusion, the present data demonstrate that the incidence of persistent candidemia was high in the VLBW infants at Liuzhou Maternity and Child Healthcare Hospital, and mechanical ventilation and intubation appeared to be the crucial factors for the development of persistent candidemia. This study highlights the importance of intensive prevention and effective treatment among neonates with persistent candidemia.

\section{Abbreviations}

95\%Cl: 95\% confidence interval; NICU: Neonatal intensive care unit; OR: Odds ratio; VLBW: Very low birth weight

\section{Acknowledgements}

Not applicable.

\section{Funding}

This manuscript was funded by Guangxi Nature Science Foundation (No. 2015GXSFBA139129), Guangxi Medical and Health Self-funding Project (No Z20170509 and No Z20180022) and the Liuzhou Science and Technology Bureau Project (No2017BD20201). The funders had no role in study design, data collection and analysis, decision to publish, or preparation of the manuscript.

\section{Availability of data and materials}

We declare that the data supporting the conclusions of this article are fully described within the article, and the database is available from the first author (fujinjianaa@126.com) upon reasonable request.

\section{Authors' contributions}

YD and JF designed the study and drafted an outline. YJ and SM participated in data analysis, JF draft of initial manuscript, YJ, SX, PQ and SM participated in diagnosed and collected the data, JF revised the manuscript and all of authors approved the final content off this manuscript.
Ethics approval and consent to participate

This study was approved by the Institutional Review Board of Liuzhou Maternity and Child Healthcare Hospital, and further permission was obtained from hospital to review the patient medical records.

\section{Consent for publication}

Not applicable.

\section{Competing interests}

The authors declare that they have no competing interests.

\section{Publisher's Note}

Springer Nature remains neutral with regard to jurisdictional claims in published maps and institutional affiliations.

\section{Author details}

'Department of Laboratory, Liuzhou Maternity and Child Healthcare Hospital, 50th Yingshan Road, Chengzhong District, Liuzhou 545001, China.

${ }^{2}$ Department of Neonatology, Liuzhou Maternity and Child Health Care Hospital, Liuzhou 545001, China.

Received: 2 August 2018 Accepted: 31 October 2018

Published online: 12 November 2018

\section{References}

1. Makhoul IR, Sujov P, Smolkin T, Lusky A, Reichman B. Epidemiological, clinical and microbiological characteristics of late-onset sepsis among very low birth weight infants in Israel: a national survey. Pediatrics. 2002;109:34-9.

2. Benjamin DK Jr, Stool BJ, Gantz MG, Walsh MC, Sánchez PJ, Das A, et al. Neonatal candidiasis: epidemiology, risk factors, and clinical judgment. Pediatrics. 2010;126:865-73.

3. Rodriguez D, Almirante B, Park BJ, Cuenca-Estrella M, Planes AM, Sanchez F, et al. Candidemia in neonatal intensive care units: Barcelona, Spain. Pediatr Infect Dis J. 2006;25:224-9.

4. Fridkin SK, Kaufman D, Edwards JR, Shetty S, Horan T. Changing incidence of Candida bloodstream infections among NICU patients in the United States: 1995-2004. Pediatrics. 2006;117:1680-7.

5. Kaufman D. Fungal infection in the very low birth weight infant. CurrOpin Infect Dis. 2004;17:253-9.

6. Leibovitz E. Neonatal candidosis: clinical picture, management controversies and consensus, and new therapeutic options. J Antimicrob Chemother. 2002;49Suppl 1:69-73.

7. Benjamin DK Jr, Stoll BJ, Fanaroff AA, McDonald SA, Oh W, Higgins RD, et al. Neonatal candidiasis among extremely low birth weight infants: risk factors, mortality rates, and neurodevelopmental outcomes at 18 to 22 months. Pediatrics. 2006;117:84-92.

8. Chang YJ, Choi IR, Shin WS, Lee JH, Kin YK, Park MS. The control of invasive Candida infection in very low birth weight infants by reduction in the use of 3rd generation cephalosporin. Korean J Pediatr. 2013;56:68-74.

9. Fu J, Ding Y, Wei B, Wang L, Xu S, Qin P, et al. Epidemiology of Candida albicans and non-C.albicans of neonatal candidemia at a tertiary care hospital in western China. BMC Infect Dis. 2017;17(1):329.

10. Celebi S, Hacimustafaoglu M, Koksal N, Ozkan H, Cetinkaya M, Ener B. Neonatal candidiasis: results of an 8 year study. Pediatrlnt. 2012;54:341-9.

11. Levy I, Shalit I, Askenazi S, Klinger G, Sirota L, Linder N. Duration and outcome of persistent Candidaemia in newborn infants. Mycoses. 2006:49:197-201.

12. Hammoud MS, Al-Taiar A, Fouad M, Raina A, Khan Z. Persistent candidemia in neonatal care units: risk factors and clinical significance. Int J Infect Dis. 2013;17:e624-8.

13. Robinson JA, Pham HD, Bloom BT, Wittler RR. Risk factors for persistent candidemia infection in a neonatal intensive care unit and its effect on mortality and length of hospitalization. J Perinatol. 2012;32:621-5.

14. Blyth CC, Chen SC, Slavin MA, Serena C, Nguyen Q, Marriott D, et al. Australian Candidemia study. Not just little adults: candidemia epidemiology, molecular characterization, and antifungal susceptibility in neonatal and pediatric patients. Pediatrics. 2009;123:1360-8.

15. Ben Abdeljelil J, Saghrouni F, Nouri S, Geith S, Khammari I, Fathallah A, et al. Neonatal invasive candidiasis in Tunisian hospital: incidence, risk factors, distribution of species and antifungal susceptibility. Mycoses. 2012;55:493-500. 
16. Liu M, Huang S, Guo L, Li H, Wang F, Zhang Ql, et al. Clinical features and risk factors for blood stream infections of Candida in neonates. ExpTher Med. 2015;10:1139-44.

17. Xia H, Wu H, Xia S, Zhu X, Chen C, Qiu G, et al. Invasive candidiasis in preterm neonates in China: a retrospective study from 11 NICUS during 2009-2011. Pediatr Infect Dis J. 2014;33:106-9.

18. Filioti J, Spiroglou K, Panteliadis CP, Roilides E. Invasive candidiasis in pediatric intensive care patients: epidemiology, risk factors, management, and outcome. Intensive Care Med. 2007;33:1272-83.

19. Ben-Ami R, Olshtain-Popsk KM, Oren I, Bishara J, Dan M, et al. Antibiotic exposure as a risk factor for fluconazole-resistant Candida bloodstream infection. Antimicrob Agents Chemother. 2012;56:2518-23.

20. Kovacicova G, Lovaszova M, Hanzen J, Roidova A, Mateicka F, Lesay M, et al. Persistent fungemia-- risk factors and outcome in 40 episodes. J Chemother. 2001:13:429-33.

21. Singhi S, Deep A. Invasive candidiasis in pediatric intensive care units. Indian J Pediatr. 2009;76:1033-44.

22. Jordan I, Balaguer M, López-Castilla JD, Belda S, Shuffelman C, Garcia-Teresa MA, et al. Per-species risk factors and predictors of invasive Candida infections in patients admitted to pediatric intensive care units. Development of ERICAP scoring systems. Pediatr Infect Dis J. 2014;33:e187-93.

23. Steinbach WJ, Roilides E, Berman D, Hoffman JA, Groll AH, Bin-Hussain I, et al. Results from a prospective, international, epidemiologic study of invasive candidiasis in children and neonates. Pediatr Infect Dis J. 2012;31:1252-7.

24. Kelly MS, Benjamin DK Jr, Smith PB. The epidemiology and diagnosis of invasive candidiasis among premature infants. ClinPerinatol. 2013;42:105-17.

25. Benjamin DK Jr, DeLong ER, Steinbach WJ, Cotton CM, Walsh TJ, Clark RH. Empirical therapy for neonatal candidemia in very low birth weight infants. Pediatrics. 2003;112:543-7.

26. Natarajan G, Lulic-Botica M, Aranda JV. Refractory neonatal candidemia and high-dose micafungin pharmacotherapy. J Perinatol. 2009;29:738-43.

27. Stoll BJ, Hansen N, Fanaroff AA, Wright LL, Carlo WA, Ehrenkranz RA, et al. Late-onset sepsis in very low birth weight neonates: the experience of the NICHD neonatal research network. Pediatrics. 2002:110:285-91.

28. Rolides E. Invasive candidiasis in neonates and children. Early Hum Dev. 2011:87 Suppl 1:S75-6.

29. Chapman RL, Faix RG. Persistently positive cultures and outcome in invasive neonatal candidiasis. Pediatr Infect Dis J. 2000;19:822-7.

30. Fisher BT, Vendetti N, Bryan M, Prasad PA, Russell Localio A, Damianos A, et al. Central venous catheter retention and mortality in children with candidemia: a retrospective cohort analysis. J Pediatric Infect Dis Soc. 2015;8:1-6.

31. Karadag-Oncel E, Kara A, Ozsurekci Y, Arikan-Akdagli S, Cengiz AB, Ceyhan $M$, et al. Candidemia in a paediatric Centre and importance of central venous catheter removal. Mycoses. 2014;58:140-8.

32. Oeser C, Lamagni T, Heath PT, Sharland M, Ladhani S. The epidemiology of neonatal and pediatric candidemia in England and Wales, 2000-2009. Pediatr Infect Dis J. 2013;32:23-6.

Ready to submit your research? Choose BMC and benefit from:

- fast, convenient online submission

- thorough peer review by experienced researchers in your field

- rapid publication on acceptance

- support for research data, including large and complex data types

- gold Open Access which fosters wider collaboration and increased citations

- maximum visibility for your research: over $100 \mathrm{M}$ website views per year

At $\mathrm{BMC}$, research is always in progress.

Learn more biomedcentral.com/submissions 